\title{
Fuzzy Support System for Total Hip Arthroplasty Stem by Ultrasonic Intraoperative Measurement
}

\author{
Naomi Yagi, ${ }^{1}$ Yutaka Hata, ${ }^{1,2}$ and Nao Shibanuma ${ }^{3}$ \\ ${ }^{1}$ Department of Electrical Engineering and Computer Sciences, Graduate School of Engineering, University of Hyogo, 2167 Shosha, \\ Himeji, Hyogo 671-2280, Japan \\ ${ }^{2}$ Division of Information Systems, WPI Immunology Frontier Research Center, Osaka University, 3-1 Yamadaoka, Suita, \\ Osaka 565-0871, Japan \\ ${ }^{3}$ Department of Orthopaedic Surgery, Kobe Kaisei Hospital, 3-11-15 Shinohara Kitamachi, Nada-Ku, Kobe, Hyogo 657-0068, Japan
}

Correspondence should be addressed to Naomi Yagi, naomi.yagi@ieee.org

Received 17 August 2012; Accepted 24 September 2012

Academic Editor: Arianna Mencattini

Copyright (C) 2012 Naomi Yagi et al. This is an open access article distributed under the Creative Commons Attribution License, which permits unrestricted use, distribution, and reproduction in any medium, provided the original work is properly cited.

\begin{abstract}
This paper describes a fuzzy system of stem implantation on total hip arthroplasty by an ultrasonic device. The system can perform automatic and accurate assessment in the surgery. In this system, we employ a single ultrasonic probe whose center frequency is $1,000 \mathrm{~Hz}$. We detect the acoustic signals when knocking the inserted stem with a hammer. We then have a correlation between the degree of tightening and the attenuation time of acoustic signal. That is, the higher tightened degree implies shorter attenuation period. The support system selects the most suitable stem size by fuzzy inference with respect to the attenuation time and its difference time from correct stem to one larger size stem which dynamically adapts to each patient. As a result, we successfully determined the suitable stem in comparison to the results of the practical surgery.
\end{abstract}

\section{Introduction}

Total hip arthroplasty (THA), which is a surgical replacement of the hip joint with an artificial prosthesis, is a reconstructive procedure that has improved the management of those diseases of the hip joint that have responded poorly to conventional medical therapy. It is an orthopedic procedure that involves the surgical excision of the head and proximal neck of the femur and removal of the acetabular cartilage and subchondral bone. This joint replacement consists of replacing a damaged joint with prosthesis. In the orthopedic surgery, the arthritic or dysfunctional joint surface is replaced with an orthopaedic prosthesis [1]. THA rebuilds the damaged hip joint with some implants and is operated to treat the hip joint diseases such as osteoarthritis. The THA implants mainly consist of cup, liner, head and stem, which play a role of acetabulum, hip cartilage, head of femur, and femur, respectively. The principal complication after THA is dislocation [2]. The primal mechanisms of dislocation are impingement of the femoral heck on the cup liner, impingement of the femur on the pelvis, and spontaneous dislocation due to excessive external force against muscular force. For example, a hip joint that is affected by osteoarthritis may be replaced entirely with a prosthetic hip. This would involve replacing the acetabulum, hip socket, and the head, and neck of the femur. The purpose of this procedure is to relieve pain, to restore range of motion, and to improve walking ability, thus leading to the improvement of muscle strength.

A joint replacement surgery is becoming a more common practice $[3,4]$. The prosthetic implant used in hip replacement consists of different parts: the acetabular cup, the femoral component, and the articular interface. The femoral component is the component that fits in the femur. The use of cementless femoral implants for total hip arthroplasty has increased in popularity over the last few decades [5]. Numerous implant designs based on different views concerning implant fixation are currently available. Bone is removed, and the femur is shaped to accept the femoral stem with attached prosthetic femoral head or ball. Options exist for different patients and indications. Correct selection of the prosthesis is important $[6,7]$. The selection of the optimum size and type of implant to fit the anatomical characteristics 


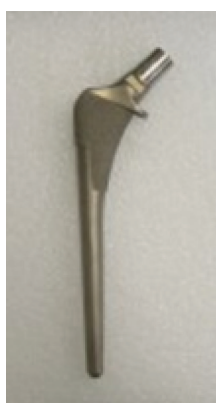

(a) Stem

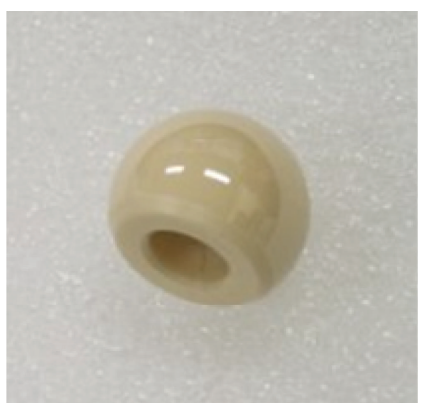

(b) Head

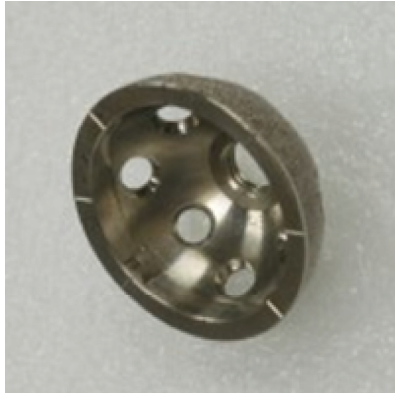

(c) Cup

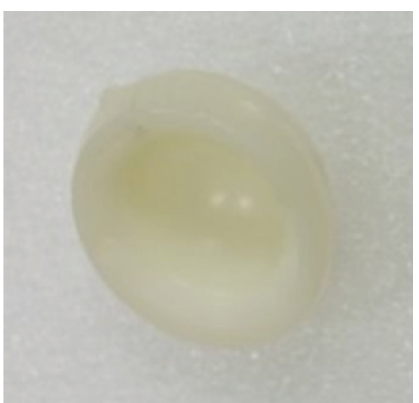

(d) Liner

FIgURE 1: Parts for total hip arthroplasty.

of the individual patient is important in order to avoid postoperative complications such as dislocation or loosening [8-10]. Regardless of implant design, primary stability of the cementless femoral implants in the femoral canal must be achieved at the time of operation and is really important for osseointegration, long-term fixation, and clinical success [11-13].

The surgeon experience in choosing the stem size to be implanted is fundamental to achieve good fixation. However, especially in the complicated cases, it still has the risk that the stem size chosen might be unsuitable. To solve the problems, it is described the mechanical stability at the time of implantation. It is difficult to predict the precise size of the implant during the preoperative plan especially when the stem implantation is planned. Thus, this system estimates the level of stability by analyzing ultrasonic wave measurements. In order to perform more quantitative measurement and more reasonable assessment based on [14-17], this paper proposes a fuzzy system of more reasonable assessment for the stem implantation on total hip arthroplasty by an ultrasonic device. In the surgery, the size of the implanted stem is decided to the surgeon after adapting to the patient from the small size stem to the larger size one in turn. It depends on the surgeon skill, and we do not have quantitative decision measures. Therefore, we propose a system which can perform automatic and accurate assessment in the surgery. It is valuable for both surgeons and patients. In this system, we employ a single ultrasonic probe whose center frequency is $1,000 \mathrm{~Hz}$. We detect the acoustic signals when knocking the inserted stem with a hammer. We then have a correlation between the degree of tightening and the attenuation time of acoustic signal, that is, the higher tightened degree implies shorter attenuation period. The stem determination support system selects the most suitable stem by fuzzy inference which dynamically adapts for each patient. As a results, we successfully determined the suitable stem in comparison to the results of the practical surgery. Consequently, we indicated the indexes in order to judge which degree the stem fits in the clinical treatment for total hip arthroplasty. Thus, we indicated how to select the most suitable stem for the patients by using the ultrasonic system. The fuzzy inference which dynamically adapts for each patient solved some problems of the individual difference. As a results, this system selected the suitable stem with higher accuracy.

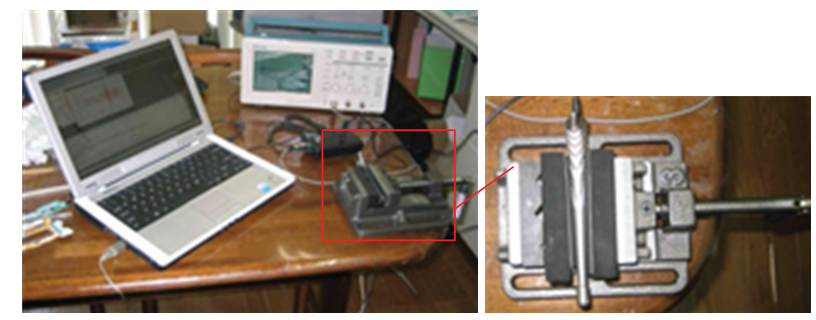

Figure 2: Preliminary experiment system.

\section{Total Hip Arthroplasty}

Hip fractures occur as a result of major or minor trauma. In elderly patients with bones weakened by osteoporosis, relatively little trauma, even walking, may result in a hip fracture. The hip is made of a ball and a socket joint, linking the dome at the head of the femur, and the cup in the pelvic bone as shown in Figure 1. A total hip prosthesis is surgically implanted to replace the damaged bone within the hip joint. Then a metal ball and a metal stem are inserted in the femur and a plastic socket is placed in the enlarged pelvis cup. The artificial components are fixed in place. The muscles and tendons are then replaced against the bones, and the incision is closed. Each patient has each various bone in the hip. So the surgeon has to find the most suitable stem for the patient while operating. In the present situation, there are some differences of selecting the suitable stem. Therefore, we propose the determination system of selecting the suitable stem for the patient.

\section{Preliminary Experiment}

We performed a preliminary experiment to analyze the degree of tightening the stem clinically measured. We confirm a correlation between the degree of tightening and the attenuation time of acoustic signal. Figure 2 shows the preliminary experiment system. The stem is fixed by the vise and a buffer material is a $20 \mathrm{~mm}$ thick sponge. We compared the difference of degree when the stem is loose and tight fixing. Figure 3 shows some sizes of stems in which the stem number is in proportion to the stem size.

As shown in Figure 4, the higher tightened degree implies shorter attenuation period. Therefore, the tightened degree 
Stem number: small

Stem size: small

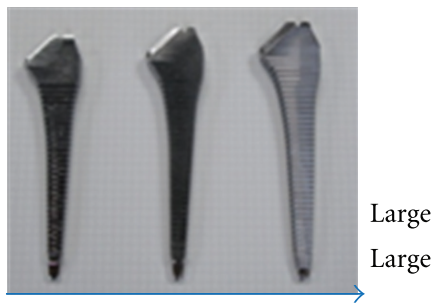

Figure 3: Stem.

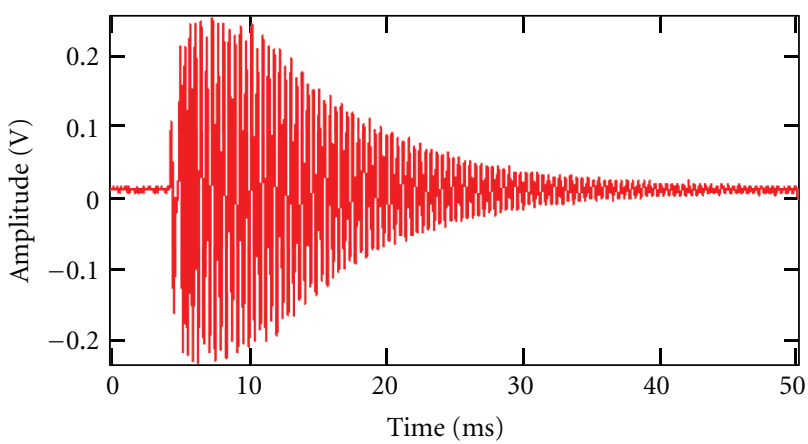

(a) Tightened degree: low

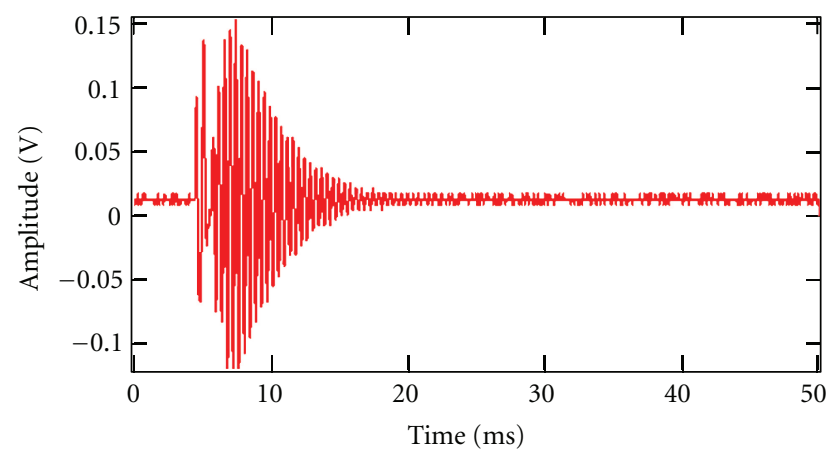

(b) Tightened degree: high

Figure 4: Attenuation time (stem number 8 ).

controls the attenuation time of amplitude [16]. In our experiment, we use three sizes of stems with number 6 , number 8 , and number 10 . By using a pressure perception film, we quantify the fixed pressures with $60 \mathrm{~N}, 80 \mathrm{~N}$, and $100 \mathrm{~N}$ as shown in Figure 5.

As a result, the changes of attenuation time in each stem are shown in Figure 6. This figure shows that the attenuation time of stem number 6 (light blue bar) and stem number 10 (light yellow bar) becomes shorter as the fixed pressure becomes stronger from $60 \mathrm{~N}$ to $100 \mathrm{~N}$. As for stem number 8, the attenuation time will shorten only when changing fixed pressure from $60 \mathrm{~N}$ to $80 \mathrm{~N}$. In the case of the same fixed pressure, the attenuation time tends to be longer as the stem size becomes larger. In the surgery, the surgeon tries to adapt for the patient from the small size stem to the larger size stem in turn. In clinical practice, the decrease of the attenuation time as the stem size becomes larger means that the fixed pressure of the stem becomes strenger. Consequently, the stem adapts better for the patient.

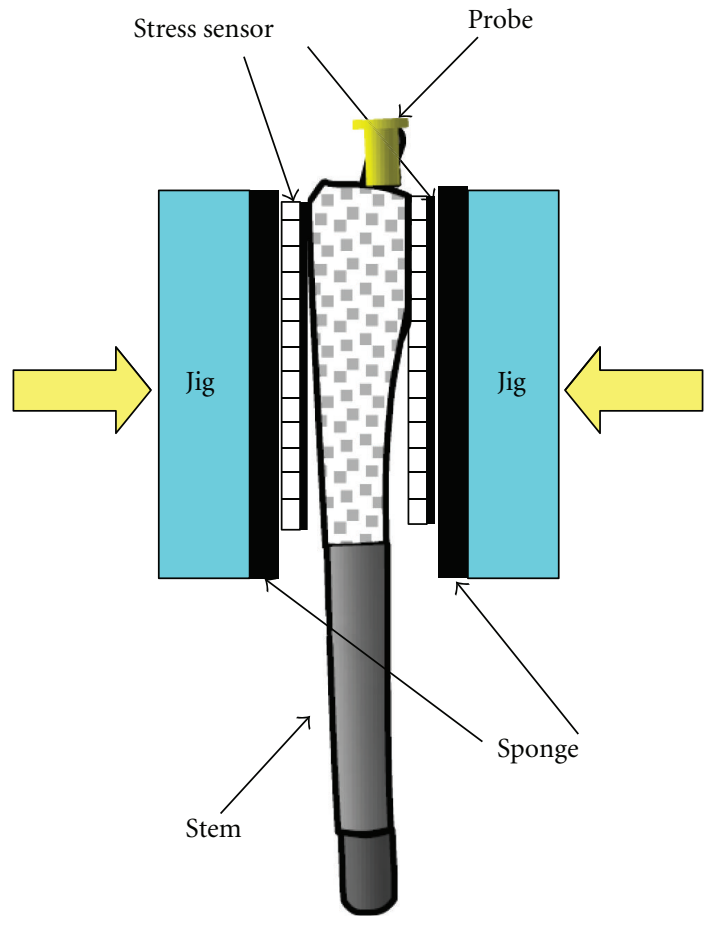

Figure 5: Pressure testing.

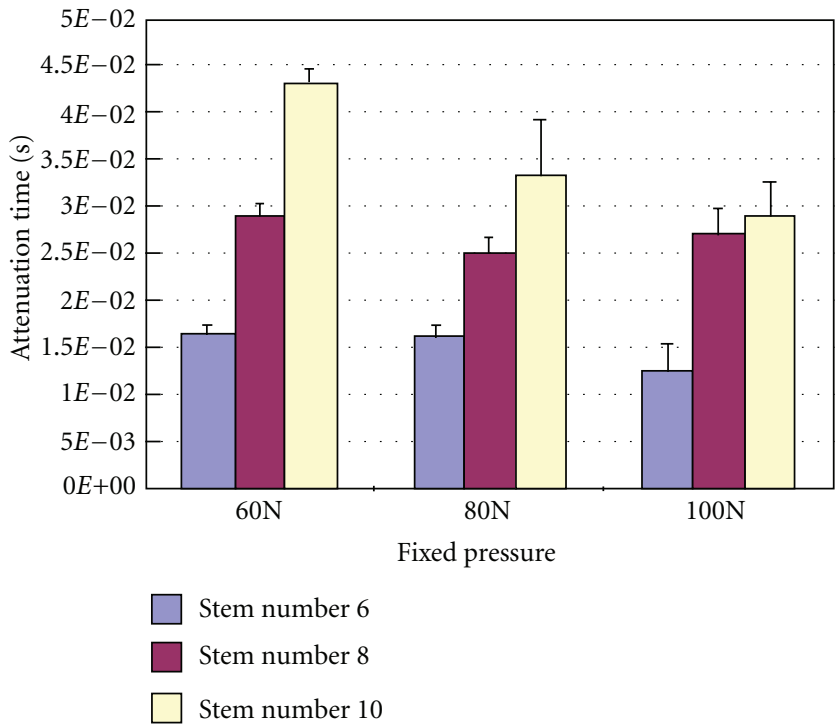

FIGURE 6: Relationship between attenuation time and pressure.

\section{Measurement System}

We employ a single ultrasonic probe whose center frequency is $1,000 \mathrm{~Hz}$ in Figure 7. The acquired ultrasonic waveform is analyzed by a personal computer [17].

Figure 8 shows the framework of this measurement system. The obtained data, attenuation time, and different time are used in fuzzy inference. After calculating the fitting rate, we select the suitable stem. By comparing it with the practical stem, we evaluate the results. 


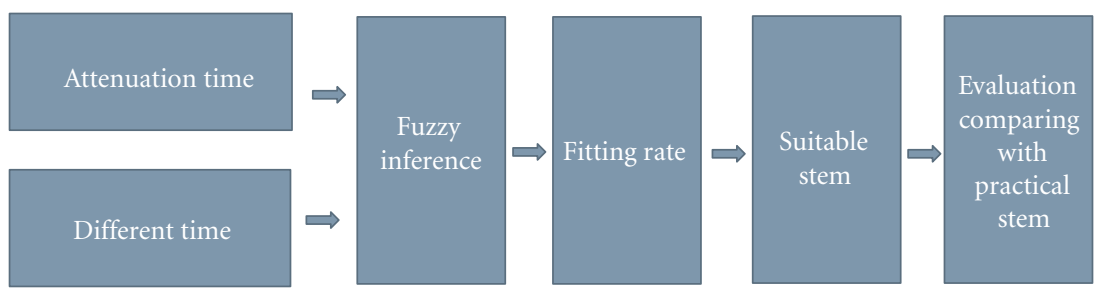

Figure 7: Framework of this system.

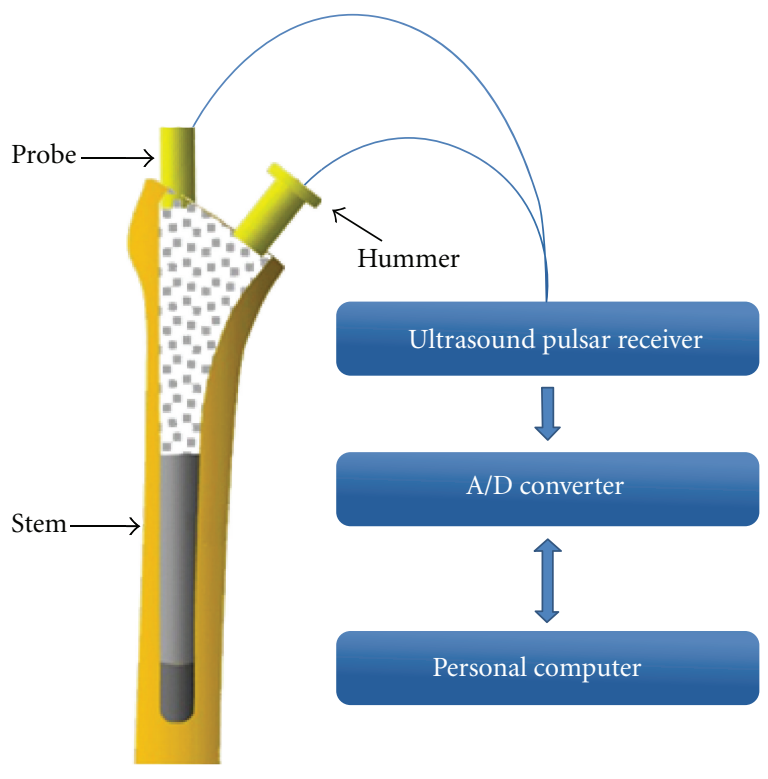

FIGURE 8: Measurement system.

At first, we make a knock to the upper point of the stem inserted with a hammer which is used as the trigger signal. By using the knocking signals, we measured the acoustic signals. The microphone for measuring $(10 \mathrm{~mm} \Phi)$ is fixed in the upper side of the stem with a built-in magnet. The acoustic data is changed to the digital data by the AD converter (Picoscope ADC-212/100) and is sent to the personal computer. In the clinical treatment, an index to judge which degree the stem fits for the patient is the knocked sound by a hammer when inserting the stem. The surgeon recognizes that the dull knocked sound means the loose fixed. From the physical opinion, in the case of loose fixing, the attenuation time of amplitude is long because the acoustic energy continues to transmit into the stem. On the other side, in the case of tight fixing, the attenuation time of amplitude is short because the acoustic energy transmits from the stem to the external fixed object and is absorbed on the early stage.

We show the flowchart of this system in Figure 9. In the surgery, the surgeons adapt the patient from the small size stem to the larger size one in turn. Firstly, we obtain the attenuation time when inserting the smallest stem. Secondly, we obtain the attenuation time and calculate the different time by using the attenuation time of the first inserted stem and one of the second inserted stems. With the fuzzy

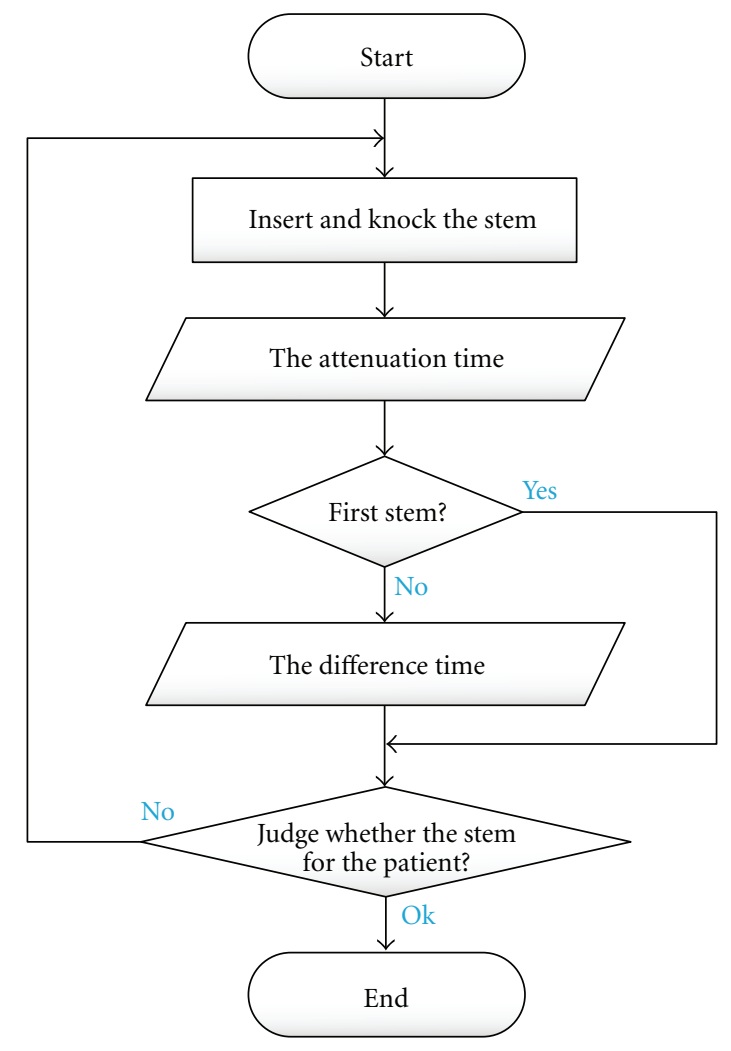

Figure 9: Flowchart.

inference, we judge whether the inserted stem is most suitable or not.

\section{Attenuation Time}

With the raw wave as shown in Figure 10, we perform envelope processing to the positive area of the acoustic wave as shown in Figure 11.

In this figure, we define $X$ as stem size (stem number $X)$ and the maximum of the attenuation value as $A_{\max }(X)$ and the time at that time as $T_{\max }(X) \cdot A_{10}(X)$ is defined as $A_{\max }(X) / 10$. We define the time $T_{10}(X)$ at the value $A_{10}(X)$.

The attenuation time $T_{\mathrm{ATT}}(X)$ of stem number $X$ is calculated by (1). We estimate the degree of fixing by using this attenuation time:

$$
T_{\mathrm{ATT}}(X)=T_{10}(X)-T_{\max }(X) .
$$




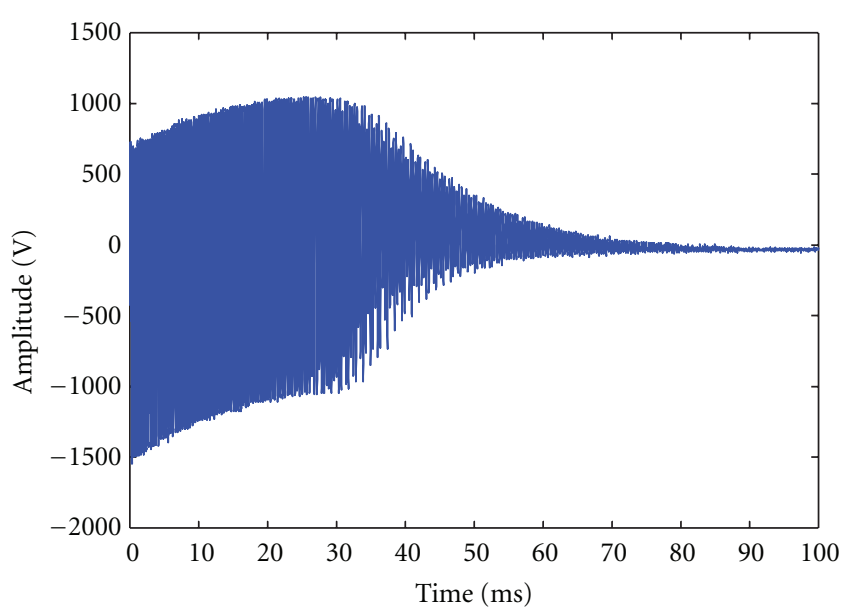

Figure 10: Raw data of acoustic wave.

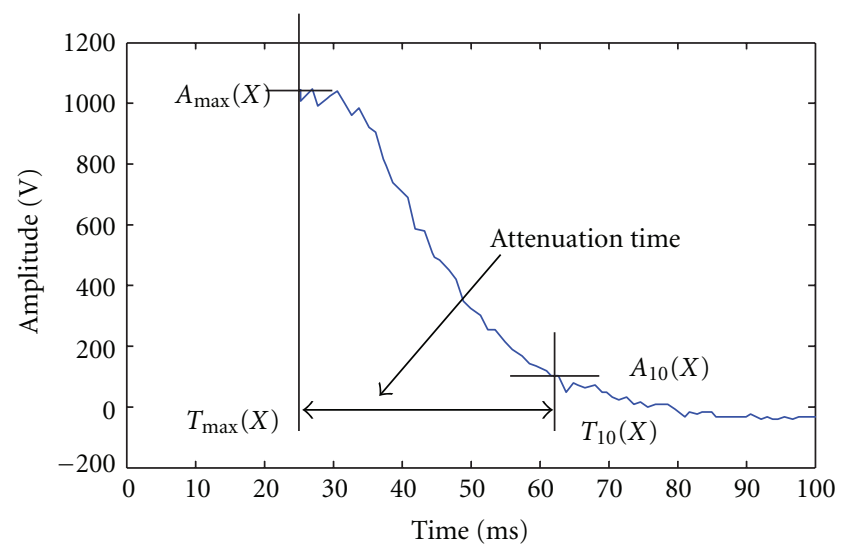

FIGURE 11: Attenuation time.

The attenuation times of stem number 4 and number 6 are shown in Figure 12. The $A_{\max }(4)$ and $A_{\max }(6)$ are of the same values. As shown in this figure, the notation $T_{\mathrm{ATT}}(4)$ is shorter than the notation $T_{\mathrm{ATT}}(6)$. This result means that stem number 4 is fixed tighter than stem number 6 .

\section{Fuzzy Inference}

We propose a fuzzy evaluation system with two features, the attenuation time $T_{\mathrm{ATT}}(X)$ and difference time for stem number $X$. The difference time $T_{\mathrm{DIF}}(X)$ is calculated by (2):

$$
T_{\mathrm{DIF}}(X)=T_{\mathrm{ATT}}(X)-T_{\mathrm{ATT}}(X-1) .
$$

The difference time is defined as the time between the attenuation time of the stem and that of the one size smaller stem. From the obtained coefficients, Knowledge 1, 2, 3, and 4 are derived.

Knowledge 1. The tight fixing has the short attenuation time.

Knowledge 2. The tight fixing has the short difference time.

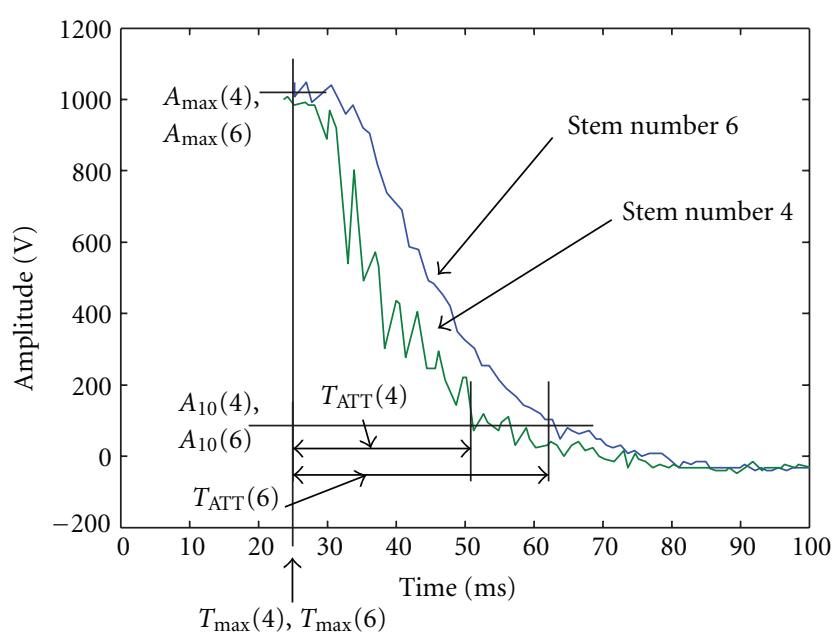

FIGURE 12: Attenuation time of stem number 4 and number 6.

Knowledge 3. The attenuation time depends on the fixed degree.

Knowledge 4. The difference time depends on the fixed degree.

These are converted to the following fuzzy if-then rules.

Rule 1. If attenuation time is short, then the degree of fixing is high.

Rule 2. If difference time is short, then the degree of fixing is high.

The fuzzy if-then rules are represented by the fuzzy membership functions as shown in Figure 13.

In this figure, the notations, $\mathrm{Th}_{a}$ and $\mathrm{Th}_{d}$, denote thresholds of the attenuation time and difference time, respectively. The input fuzzy membership functions are defined by the means and the standard deviations (SDs) of the attenuation time and the difference time, respectively.

The notation $\mathrm{Th}_{a}$ is determined as the time which is the standard deviation being shorter than the mean of the attenuation time. The notation $\mathrm{Th}_{d}$ is determined as the time which is the standard deviation being shorter than the mean of the difference time. Two fuzzy degrees, $\mu_{\mathrm{ATT}}(X)$ and $\mu_{\mathrm{DIF}}(X)$, are calculated by (3):

$$
\begin{aligned}
& \mu_{\mathrm{ATT}}(X)=\min \left(S_{T_{\mathrm{ATT}}}(t), \mathrm{HIGH}_{a}\right), \\
& \mu_{\mathrm{DIF}}(X)=\min \left(S_{T_{\mathrm{DIF}}}(t), \mathrm{HIGH}_{d}\right) .
\end{aligned}
$$

The fuzzy singleton functions, $S_{T_{\mathrm{ATT}}}(t)$ and $S_{T_{\mathrm{DIF}}}(t)$, are defined by (4):

$$
\begin{aligned}
& S_{T_{\mathrm{ATT}}}(t)= \begin{cases}1, & \text { if } t=T_{\mathrm{ATT}}(X), \\
0, & \text { otherwise }\end{cases} \\
& S_{T_{\mathrm{DIF}}}(t)= \begin{cases}1, & \text { if } t=T_{\mathrm{DIF}}(X), \\
0, & \text { otherwise }\end{cases}
\end{aligned}
$$




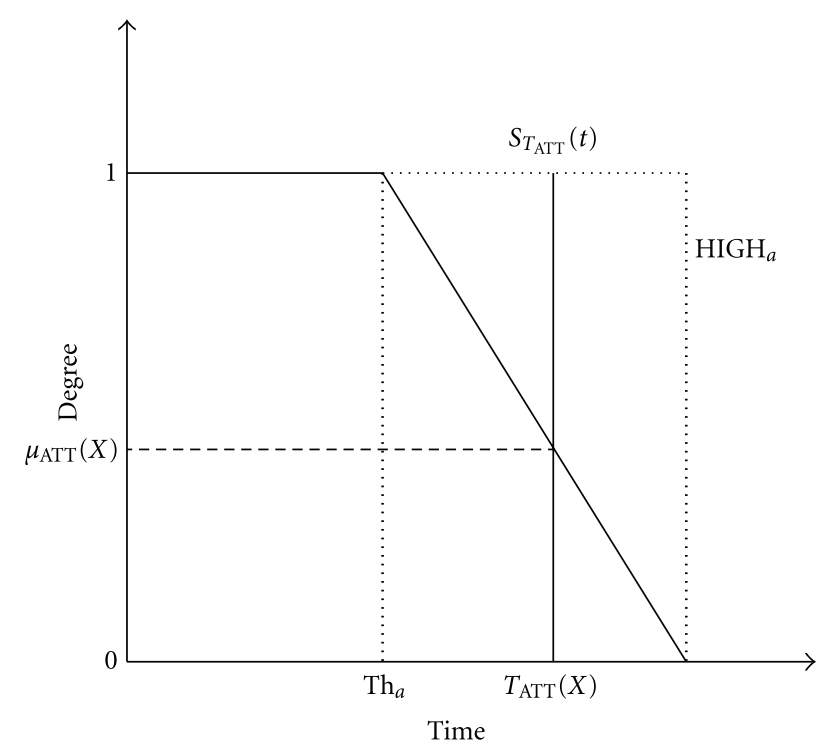

(a) Attenuation time

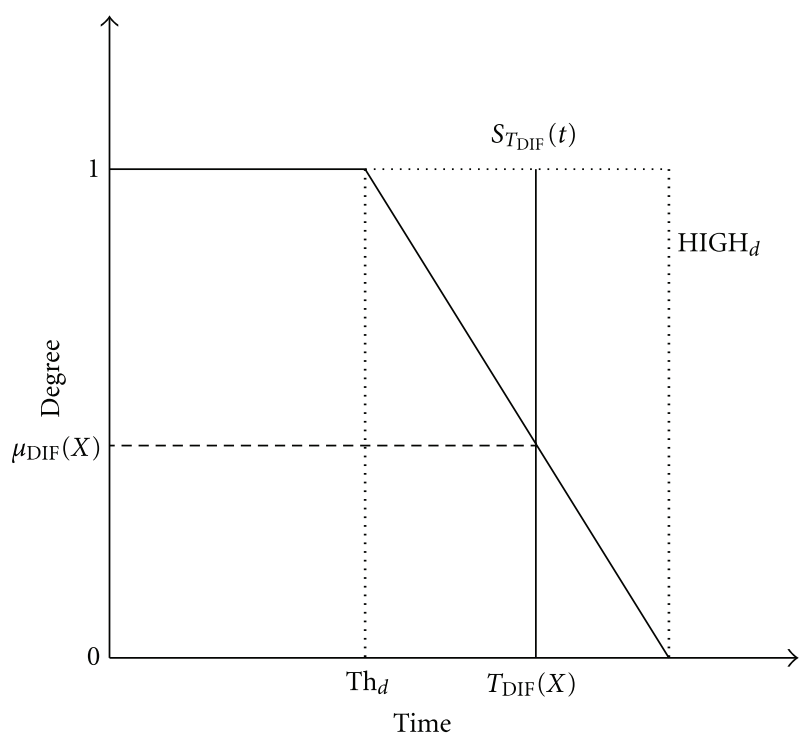

(b) Difference time

FIGURE 13: Fuzzy membership function.

We calculate the fixed degree $\mu_{\mathrm{FIX}}(X)$ of stem number $X$ by (5):

$$
\mu_{\mathrm{FIX}}(X)=r \times \mu_{\mathrm{ATT}}(X)+(1-r) \times \mu_{\mathrm{DIF}}(X),
$$

where the notation, $r$, is the ratio; it is experimentally decided.

\section{Experimental Results}

Under consideration of the thresholds in Tables 1 and 2, the threshold of the attenuation time, $\mathrm{Th}_{a}$, is more adaptable than that of the difference time, $\mathrm{Th}_{d}$. Therefore, we calculate the adaptable ratio $(r=0.823)$ and put the adaptable ratio

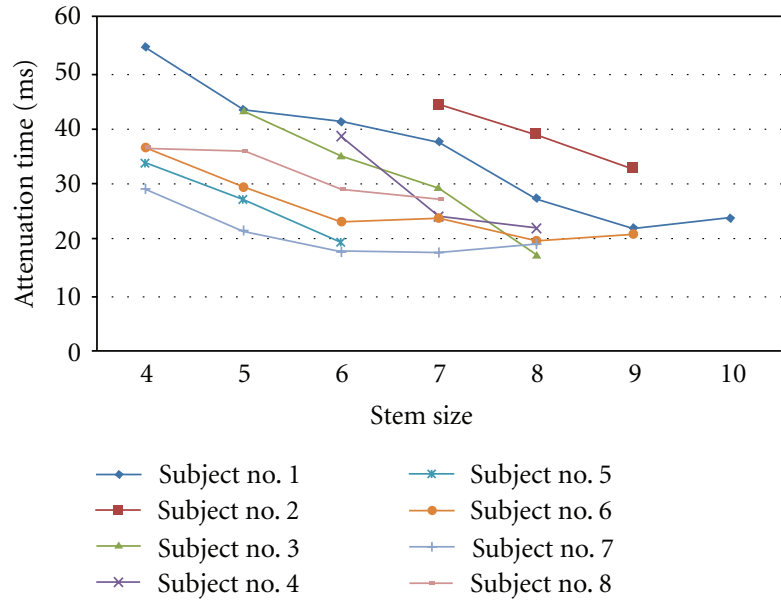

Figure 14: Selected stem size in practical surgery.

to the fuzzy membership functions. The total degree $\mu_{\mathrm{FIX}}(X)$ is calculated by (6):

$$
\mu_{\mathrm{FIX}}(X)=\mu_{\mathrm{ATT}}(X) \times 0.823+\mu_{\mathrm{DIF}}(X) \times(1-0.823) .
$$

We applied our proposed system to 8 total hip arthroplasty patients. The result of the fixed stem size and the analyzed stem size was shown in Table 3 . The attenuation time measured in the practical surgery is shown in Figure 14. In this figure, the horizontal axis means the size of the stem and the vertical axis means the attenuation time.

By using our proposed method, we selected the suitable stem of 7 patients. Concerning subject number 1 and number 7 , we selected two sizes of the determined stem. Because these subjects have the fixed stem which is one size larger than the stem with the shortest attenuation time, it is left out of consideration that the determined stem has two types which are the fixed size stem and the one size smaller stem. Concerning subject number 2 and number 5 , we did not select the suitable stem because the difference time is relatively large as shown in Figure 14. As a result, the effectiveness of selecting the suitable stem was $87.5 \%$.

\section{Conclusion}

In this paper, we have proposed a stem determination system for total hip arthroplasty patients. The acquired ultrasonic waveform with the single ultrasonic probe with the center frequency $1,000 \mathrm{~Hz}$ is analyzed by a personal computer. At first, we make a knock to the upper point of the stem inserted with a hammer which has the trigger signals. By using the knocking signals, we measured the acoustic signals. The ultrasonic probe for measuring is fixed in the upper side of the stem with a built-in magnet. The acoustic data is changed to the digital data by the $\mathrm{AD}$ converter and is sent to the personal computer. In the surgery, the surgeon tries to adapt for the patient from the small size stem to the larger size stem in turn. Therefore, our system which selects the best suitable stem by using the attenuation time is valuable for surgeons and patients of total hip arthroplasty. 
TABLE 1: Threshold of fuzzy membership function (attenuation time).

\begin{tabular}{|c|c|c|c|c|c|c|c|c|}
\hline $\mathrm{Th}_{a}$ & $\begin{array}{c}\text { Subject } \\
\text { number } 1\end{array}$ & $\begin{array}{c}\text { Subject } \\
\text { number } 2\end{array}$ & $\begin{array}{c}\text { Subject } \\
\text { number } 3\end{array}$ & $\begin{array}{c}\text { Subject } \\
\text { number } 4\end{array}$ & $\begin{array}{c}\text { Subject } \\
\text { number } 5\end{array}$ & $\begin{array}{c}\text { Subject } \\
\text { number } 6\end{array}$ & $\begin{array}{c}\text { Subject } \\
\text { number } 7\end{array}$ & $\begin{array}{c}\text { Subject } \\
\text { number } 8\end{array}$ \\
\hline Stem number 4 & 30.61 & - & - & - & 28.44 & 27.50 & 30.94 & 27.50 \\
\hline Stem number 5 & 23.22 & - & 23.19 & - & 25.48 & 24.58 & 28.42 & 23.19 \\
\hline Stem number 6 & 18.91 & - & 18.53 & 18.60 & 21.88 & 20.51 & 22.59 & 19.13 \\
\hline Stem number 7 & 18.91 & 20.13 & 19.41 & 20.52 & - & 20.64 & 23.04 & 19.82 \\
\hline Stem number 8 & 14.75 & 17.42 & 17.30 & 15.74 & - & 16.39 & 16.65 & - \\
\hline Stem number 9 & 18.51 & 20.79 & - & - & - & 19.72 & - & - \\
\hline Stem number 10 & - & - & - & - & - & - & - & - \\
\hline
\end{tabular}

TABLE 2: Threshold of fuzzy membership function (different time).

\begin{tabular}{|c|c|c|c|c|c|c|c|c|}
\hline $\mathrm{Th}_{d}$ & $\begin{array}{c}\text { Subject } \\
\text { number } 1\end{array}$ & $\begin{array}{c}\text { Subject } \\
\text { number } 2\end{array}$ & $\begin{array}{c}\text { Subject } \\
\text { number } 3\end{array}$ & $\begin{array}{c}\text { Subject } \\
\text { number } 4\end{array}$ & $\begin{array}{c}\text { Subject } \\
\text { number } 5\end{array}$ & $\begin{array}{c}\text { Subject } \\
\text { number } 6\end{array}$ & $\begin{array}{c}\text { Subject } \\
\text { number } 7\end{array}$ & $\begin{array}{c}\text { Subject } \\
\text { number } 8\end{array}$ \\
\hline Stem number 4 & - & - & - & - & - & - & - & - \\
\hline Stem number 5 & 2.21 & - & - & - & 2.17 & 2.15 & 2.01 & 6.04 \\
\hline Stem number 6 & 4.73 & - & 2.95 & - & 2.93 & 3.00 & 3.80 & 2.95 \\
\hline Stem number 7 & -1.73 & - & -2.09 & -0.38 & - & -0.27 & -0.62 & -1.27 \\
\hline Stem number 8 & -0.41 & -0.08 & -0.13 & 0.73 & - & 0.19 & 2.69 & - \\
\hline Stem number 9 & -2.57 & -2.45 & - & - & - & 5.28 & - & - \\
\hline Stem number 10 & - & - & - & - & - & - & - & - \\
\hline
\end{tabular}

TABLe 3: Result of stem size.

\begin{tabular}{lcc}
\hline & Determined size & Used size \\
\hline Subject number 1 & 9 or 10 & 10 \\
Subject number 2 & N/A & 9 \\
Subject number 3 & 8 & 8 \\
Subject number 4 & 8 & 8 \\
Subject number 5 & 5 or 6 & 6 \\
Subject number 6 & 9 & 9 \\
Subject number 7 & 7 or 8 & 8 \\
Subject number 8 & 7 & 7 \\
\hline
\end{tabular}

As a result, we successfully determined the suitable stem in comparison to the results obtained from the practical surgery. It became an index in order to judge which degree the stem fits in the clinical treatment for total hip arthroplasty. It remains as future works to consider the much better effective method and to apply the method to total hip arthroplasty patients.

\section{References}

[1] A. Sansom, J. Donovan, C. Sanders et al., "Routes to total joint replacement surgery: patients' and clinicians' perceptions of need," Arthritis Care and Research, vol. 62, no. 9, pp. 12521257, 2010.

[2] G. E. Lewinnek, J. L. Lewis, and R. Tarr, "Dislocations after total hip-replacement arthroplasties," Journal of Bone and Joint Surgery A, vol. 60, no. 2, pp. 217-220, 1978.

[3] W. J. Maloney, T. Schmalzried, and W. H. Harris, "Analysis of long-term cemented total hip arthroplasty retrievals," Clinical Orthopaedics and Related Research, no. 405, pp. 70-78, 2002.
[4] E. Schneider, C. Kinast, J. Eulenberger, D. Wyder, G. Eskilsson, and S. M. Perren, "A comparitive study of the initial stability of cementless hip prostheses," Clinical Orthopaedics and Related Research, no. 248, pp. 200-209, 1989.

[5] R. K. Sinha, D. S. Dungy, and H. B. Yeon, "Primary total hip arthroplasty with a proximally porous-coated femoral stem," Journal of Bone and Joint Surgery A, vol. 86, no. 6, pp. 12541261, 2004.

[6] M. Lannocca, E. Varini, A. Cappello, L. Cristofolini, and E. Bialoblocka, "Intra-operative evaluation of cementless hip implant stability: a prototype device based on vibration analysis," Medical Engineering and Physics, vol. 29, no. 8, pp. 886-894, 2007.

[7] E. Varini, L. Cristofolini, F. Traina, M. Viceconti, and A. Toni, "Can the rasp be used to predict intra-operatively the primary stability that can be achieved by press-fitting the stem in cementless hip arthroplasty?" Clinical Biomechanics, vol. 23, no. 4, pp. 408-414, 2008.

[8] D. D. D'Lima, A. G. Urquhart, K. O. Buehler, R. H. Walker, and C. W. Colwell, "The effect of the orientation of the acetabular and femoral components on the range of motion of the hip at different head-neck ratios," Journal of Bone and Joint Surgery A, vol. 82, no. 3, pp. 315-321, 2000.

[9] S. L. Delp, A. V. Komattu, and R. L. Wixson, "Superior displacement of the hip in total joint replacement: effects of prosthetic neck length, neck-stem angle, and anteversion angle on the moment- generating capacity of the muscles," Journal of Orthopaedic Research, vol. 12, no. 6, pp. 860-870, 1994.

[10] F. Pierchon, G. Pasquier, A. Cotten, C. Fontaine, J. Clarisse, and A. Duquennoy, "Causes of dislocation of total hip arthroplasty. CT study of component alignment," Journal of Bone and Joint Surgery B, vol. 76, no. 1, pp. 45-48, 1994.

[11] T. Albrektsson, P. I. Branemark, H. A. Hansson, and J. Lindstrom, "Osseointegrated titanium implants. Requirements for ensuring a long-lasting, direct bone-to-implant anchorage in 
man," Acta Orthopaedica Scandinavica, vol. 52, no. 2, pp. 155$170,1981$.

[12] J. J. Callaghan, C. S. Fulghum, R. R. Glisson, and S. K. Stranne, "The effect of femoral stem geometry on interface motion in uncemented porous-coated total hip prostheses. Comparison of straight-stem and curved- stem designs," Journal of Bone and Joint Surgery A, vol. 74, no. 6, pp. 839-848, 1992.

[13] P. S. Nourbash and W. G. Paprosky, "Cementless femoral design concerns: rationale for extensive porous coating," Clinical Orthopaedics and Related Research, no. 355, pp. 189-199, 1998.

[14] N. Shibanuma, K. Nagamune, Y. Hata, T. Nishiyama, H. Tateishi, and M. Kurosaka, "Evaluation of the stability of a femoral stem using ultrasound," in Proceedings of the 53rd Annual Meeting of the Orthopaedic Research Society, p. 1733, 2007.

[15] N. Shibanuma, Y. Hata, T. Nishiyama, T. Fujishiro, H. Tateishi, and M. Kurosaka, "Determination of total hip arthroplasty stem stability by intraoperative measurement using an acoustic testing technique," in Proceedings of the 54rd Annual Meeting of the Orthopaedic Research Society, p. 1842, 2008.

[16] N. Yagi, Y. Oshiro, O. Ishikawa, Y. Hata, and N. Shibanuma, "Estimation system for total hip arthroplasty by acoustic signal," in Proceedings of the IEEE Workshop on Robotic Intelligence in Informationally Structured Space (RIISS '11), IEEE Symposium Series on Computational Intelligence, pp. 32-36, April 2011.

[17] N. Yagi, Y. Oshiro, O. Ishikawa, Y. Hata, and N. Shibanuma, "Fuzzy RASP determination by $1 \mathrm{kHz}$ ultrasonic probe for total hip arthroplasty," in Proceedings of the International Conference on Fuzzy Systems, pp. 1017-1021, 2011. 

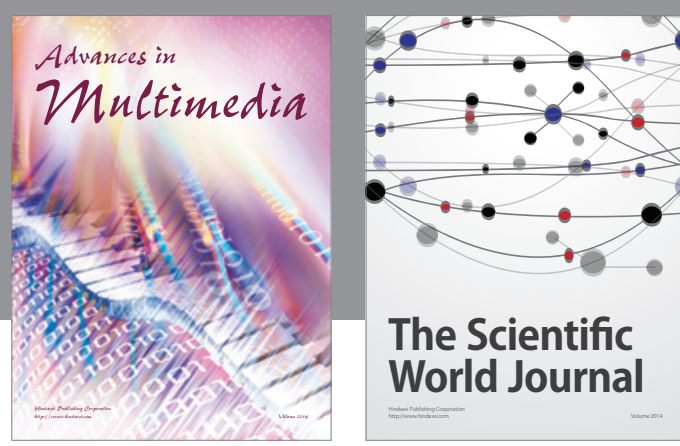

The Scientific World Journal
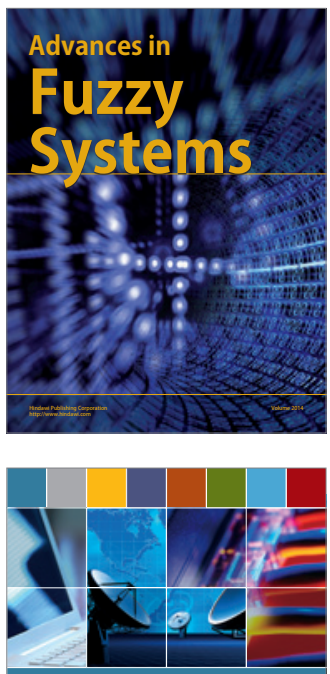

Computer Networks and Communications
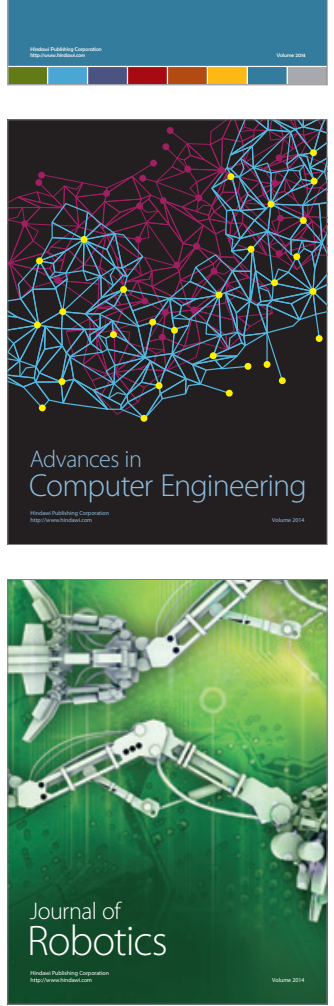
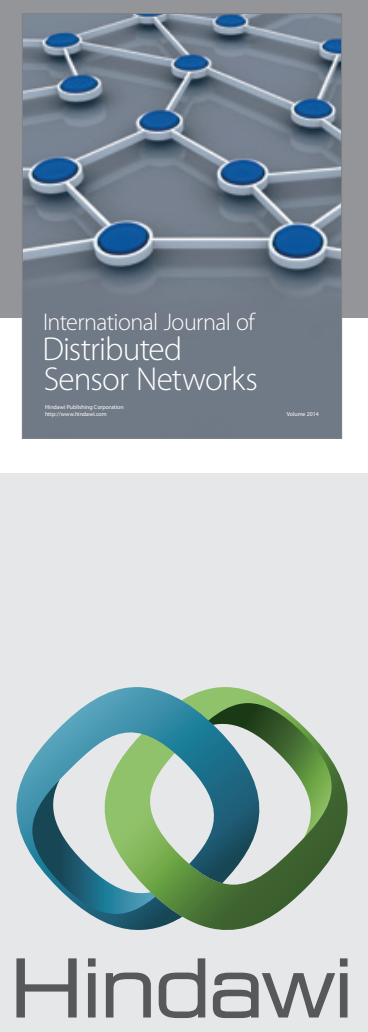

Submit your manuscripts at

http://www.hindawi.com
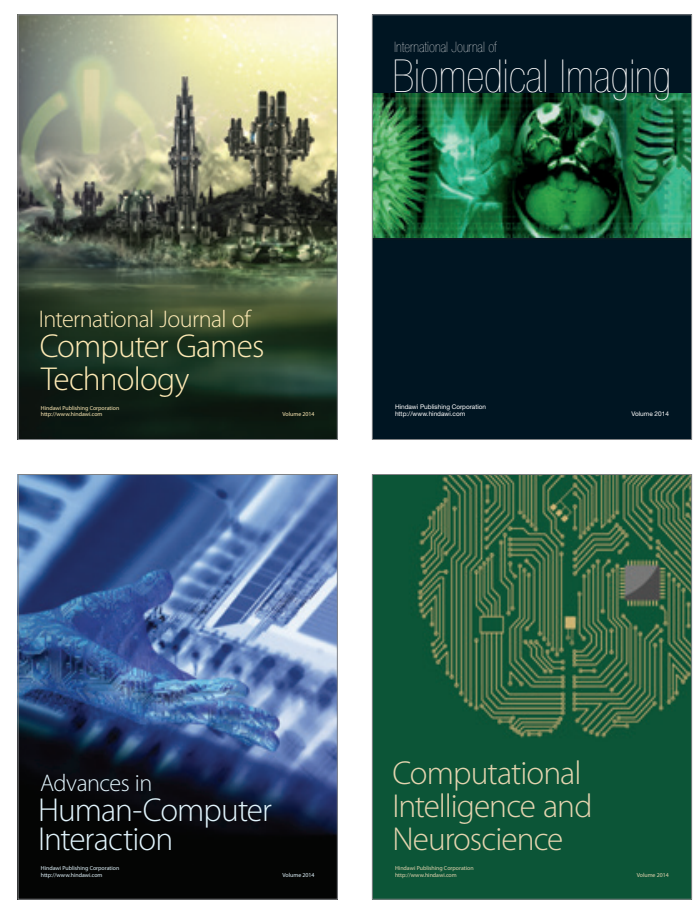
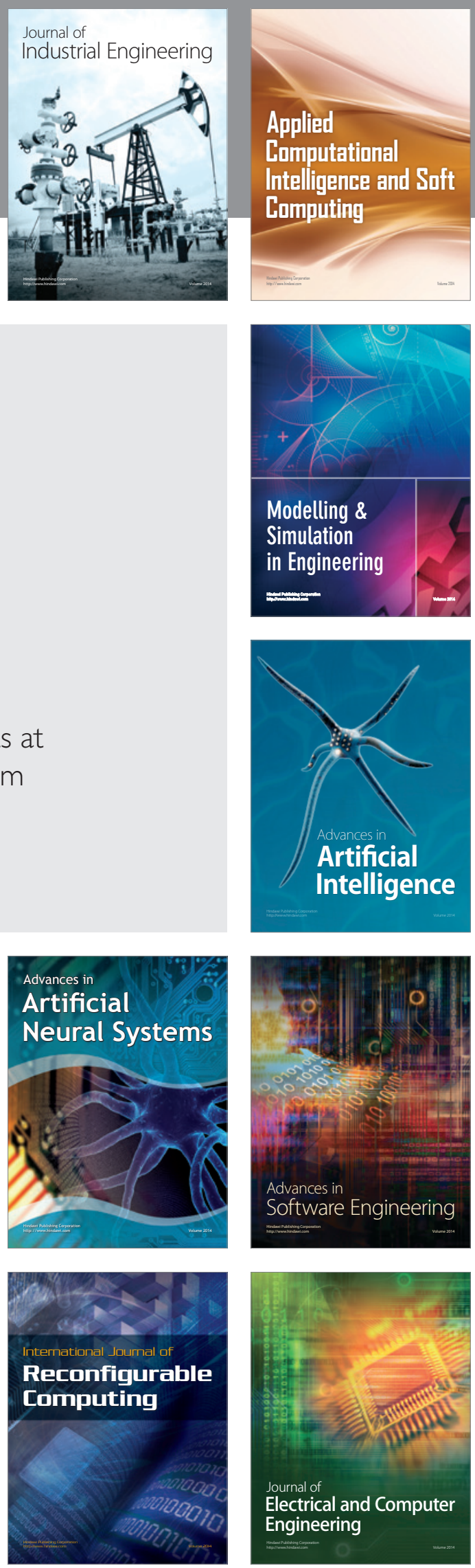